\title{
Biogeochemical Oceanographic Data Assimilation: Dimensionality Reduced Kalman Filter \\ For Mediterranean Sea Forecasting
}

\section{S. Spada ${ }^{1,2}$, G.Cossarini1 ${ }^{1}$, S. Salon ${ }^{1}$ \& S. Maset $^{2}$}

${ }^{1}$ Istituto Nazionale di Oceanografia e di Geofisica Sperimentale, Trieste, Italy

${ }^{2}$ Università degli studi di Trieste, Trieste, Italy

simonespada.info@gmail.com

sspadaeinogs.it

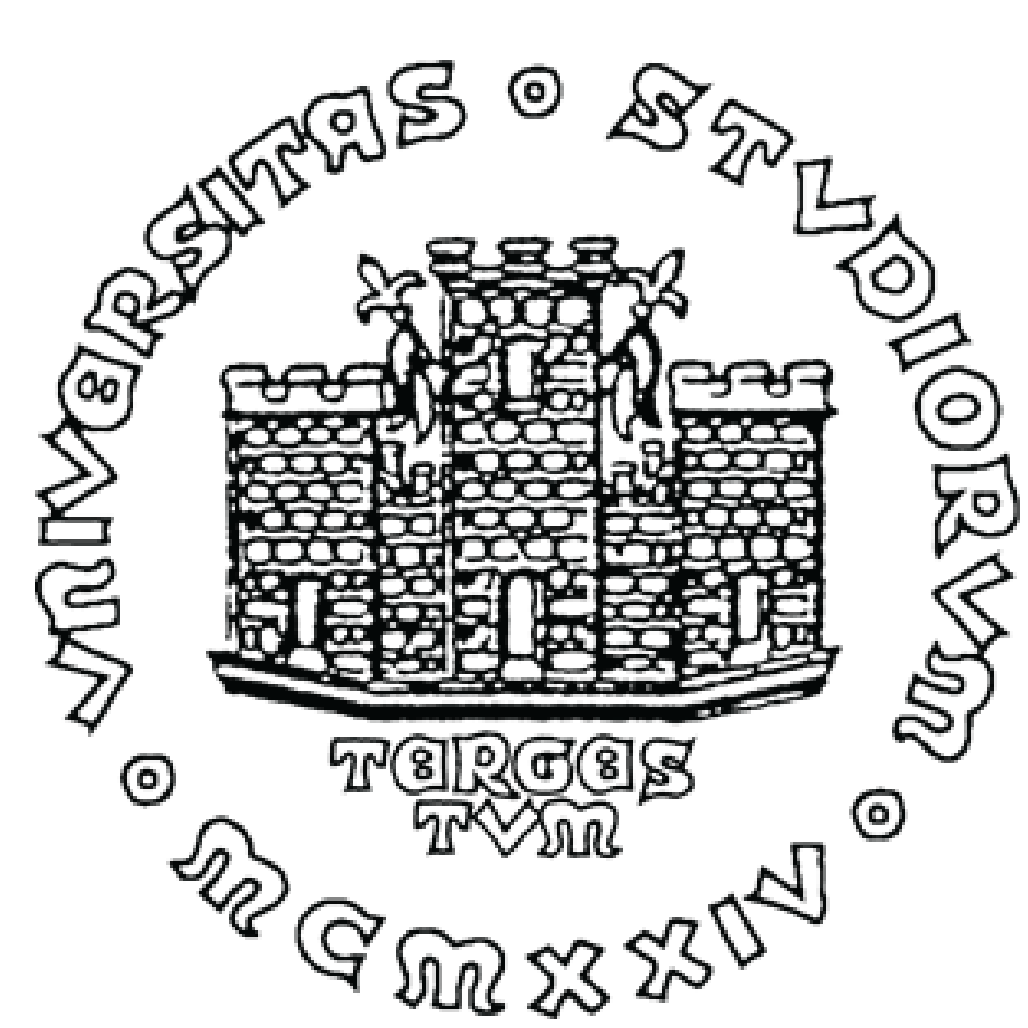

Abstract

Data assimilation is a key element to improve the performance of biogeochemical ocean/marine forecasting systems. Handling the very big dimension of the state vector of the system (often of the order of $10^{6}$ ) remains an issue, also considerin he computational efficiency of operational systems. Indeed, simple product operations involving he in litance natrices are complexity of this task, often adding strong hypotheses to simplify the problem and decrease the computational cost.

The MedBFM model system ([5], [8], and references thereby), which is responsible for monitoring and forecasting the biogeochemical state of the Mediterranean Sea within the European Copernicus Marine Services (see http: / /marine, coperni poses the background error covariance matrix into sequential operators to reduce complexity [8].

In this work, we developed a novel Kalman Filter for the MedBFM system. The novel Kalman Filter scheme starts from SEIK ([9]) approach but benefits from advanced Principal Component Analysis (PCA, [4]) to reduce the dimension of covariance matrices and improve the computational efficiency.

We compared the standard SEIK filter and the new Kalman filter implementations in a one dimensional transport model with 2 biological variables in terms of root mean square distance. In the vast majority of the experiments, the new Kalman filter performances.

\section{The SEIK Filter}

The notation mostly follows [7]

Background state:

Background error covariance:

Model error covariance:

Observation:

Observation error covariance:

Perturbation matrix:

Matrix of ensemble of $N$ members:

Evolution of the ensemble:

Observation operator on the columns of $X^{f}$

Error subspace basis:

Forecast

Forecast covariance:

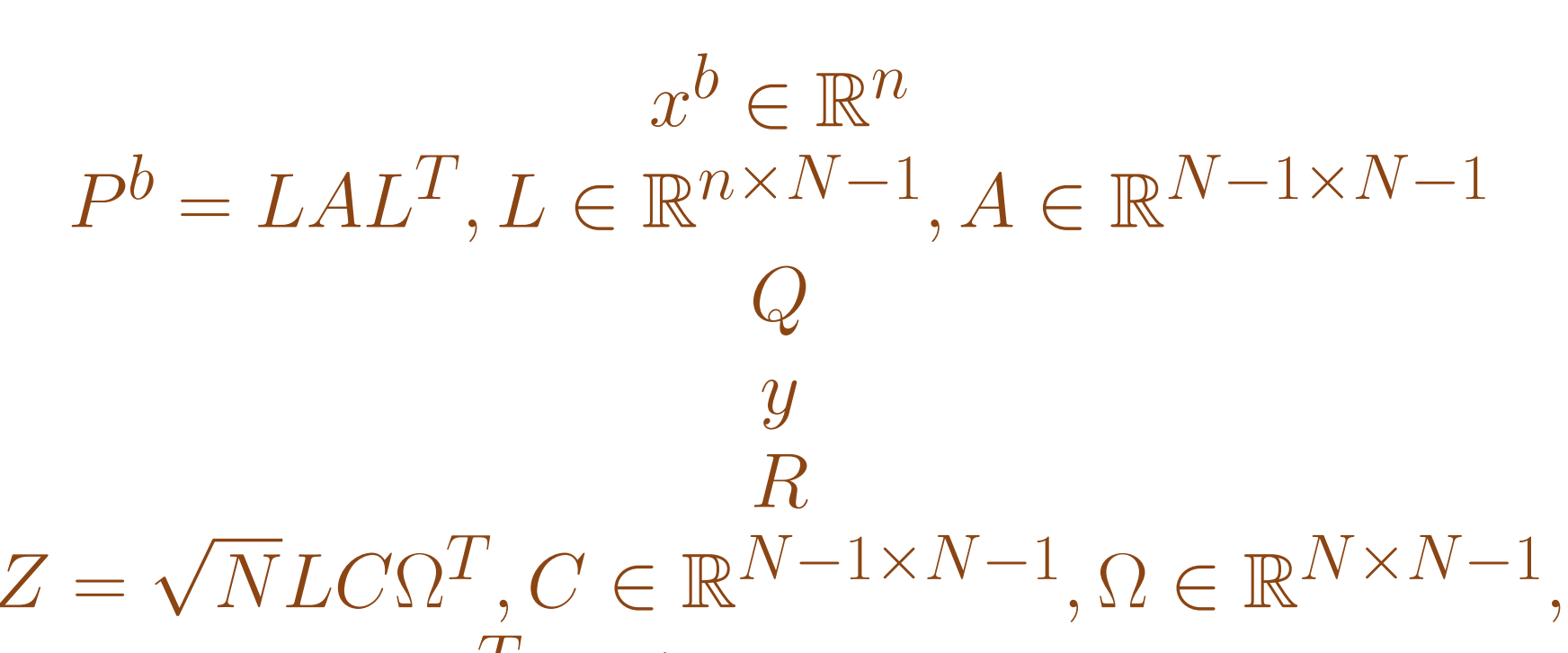

with $C C^{T}=A$ and $\Omega$ random matrix

with orthonoral columns and zero columns sum $X=x^{b} \mathbb{1}_{1 \times N}+Z$

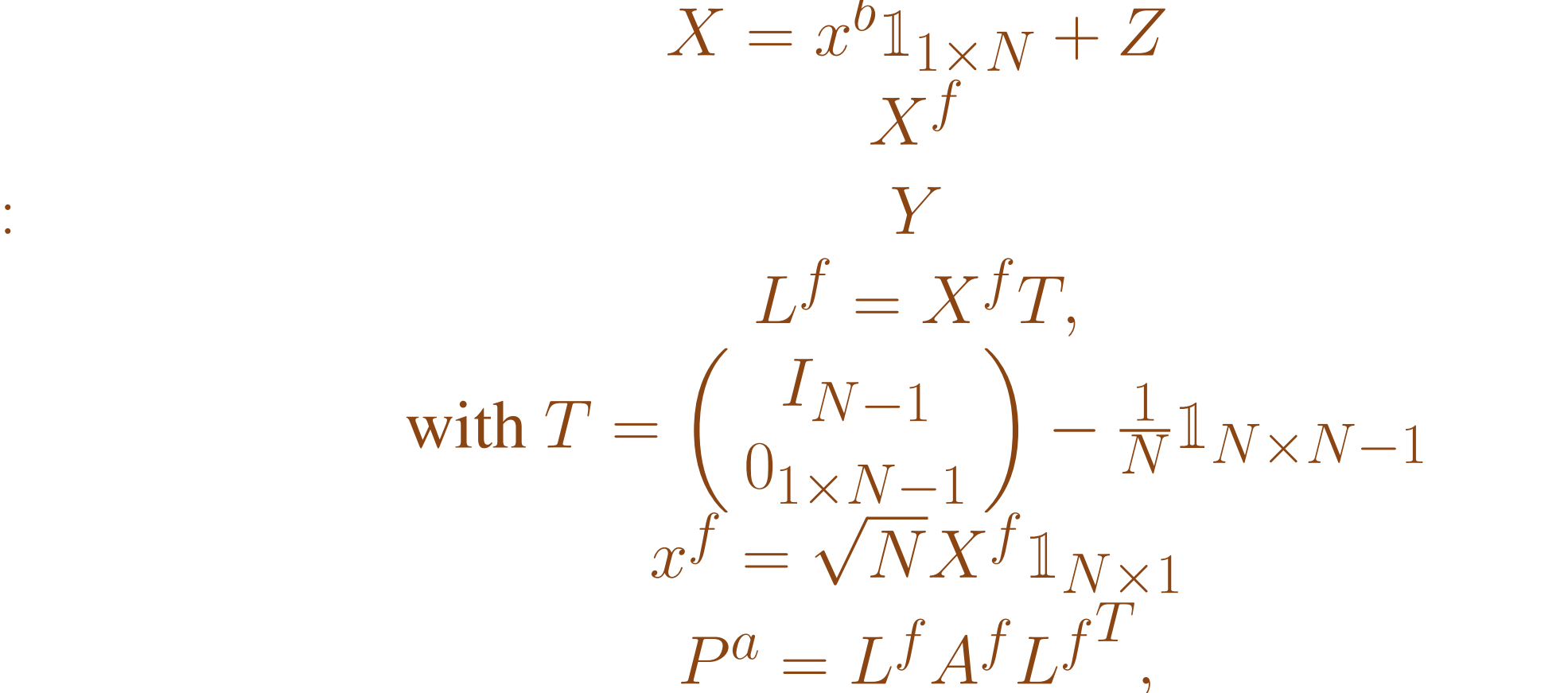

Analysis covariance:

with $A^{f}=\frac{1}{N}\left(T^{T} T\right)^{-1}+\left(L^{f^{T}} L^{f}\right)^{-1} L^{f^{T}} Q L^{f}\left(L^{f^{T}} L^{f}\right)^{-1}$

Analysis:

with $A^{a-1}=A^{f^{-1}}+(Y T)^{T} R^{-1} Y T$

$x^{a}=x^{f}+L^{f} A^{a}(Y T)^{T} R^{-1}\left(y-y^{f}\right)$

with $y^{f}$ the observation operator on $x^{f}$

The SEIK+PCA Filter

$Z$ is substituted by

\section{$\bar{Z}=\sqrt{N} U_{r} S_{r} \Omega_{r}^{T}$}

$$
U S V^{T}=L C
$$

is the singular values decomposition of $L C$.

If $r$ singular values are predominant (with $2 r>N-1$ ), then $U_{r} \in \mathbb{R}^{n \times r}$ and $S_{r} \in \mathbb{R}^{r \times r}$ are the submatrices of $U$ and $S$ that refer to those values.

$\Omega_{r} \in \mathbb{R}^{r \times N}$ has the same properties as $\Omega$, but it is obtained completing the matrix

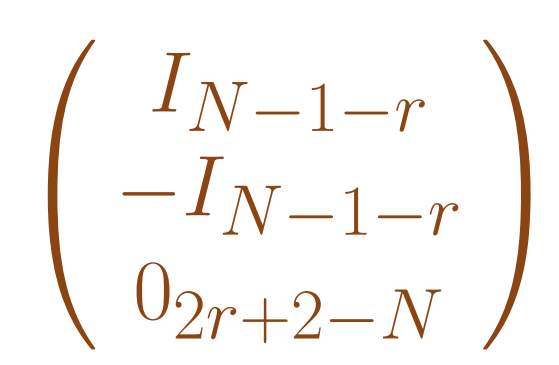

\section{The experiment}

The filters has been compared in a one dimensional transport model with 2 biological variables.

One variable provided data in a limited part of the domain (Assimilation type 1: only in the center, assimilation type 2: uniformly distributed points) every 20 timesteps, while the second variable was hidden. 20 different parameter sets has been tried for each case.

The model

$$
\begin{gathered}
d_{p}=A c_{p}\left(M-c_{p}\right)-B c_{p} c_{q}, \\
d_{q}=C c_{p} c_{q}-D c_{p}, \\
\frac{\partial}{\partial t} c_{i}=-V(x) \frac{\partial}{\partial x} c_{i}+K \frac{\partial^{2}}{\partial x^{2}} c_{i}, \quad \forall i \in\{p, q\} .
\end{gathered}
$$

Results

$\begin{array}{llllllllll}\text { Ex1 } & \text { Ex2 } & \text { Ex3 } & \text { Ex4 } & \text { Ex5 } & \text { Ex6 } & \text { Ex7 } & \text { Ex8 } & \text { Ex9 } & \text { Ex10 }\end{array}$

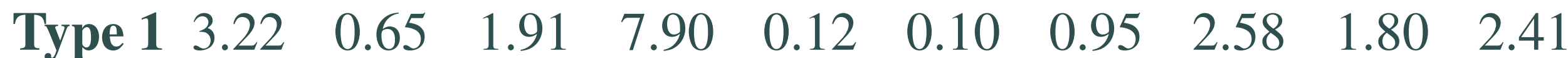
\begin{tabular}{lllllllllll} 
Type 2 & 0.80 & 0.22 & 0.03 & -0.18 & 0.09 & 0.26 & 0.02 & 1.20 & 1.47 & 1.14 \\
\hline
\end{tabular} Ex11 Ex12 Ex13 Ex14 Ex15 Ex16 Ex17 Ex18 Ex19 Ex20 $\begin{array}{llllllllllll}\text { Type } 1 & 0.86 & 7.57 & 0.86 & 3.89 & -1.14 & 3.74 & 0.54 & 2.46 & 1.34 & 0.06\end{array}$ $\begin{array}{lllllllllll}\text { Type } 2 & 1.14 & 1.51 & 0.06 & -0.09 & 0.35 & 0.43 & 0.09 & 0.43 & -0.29 & 1.30\end{array}$

Table 1: Difference of root mean square distance between the classic and the modified SEIK filter

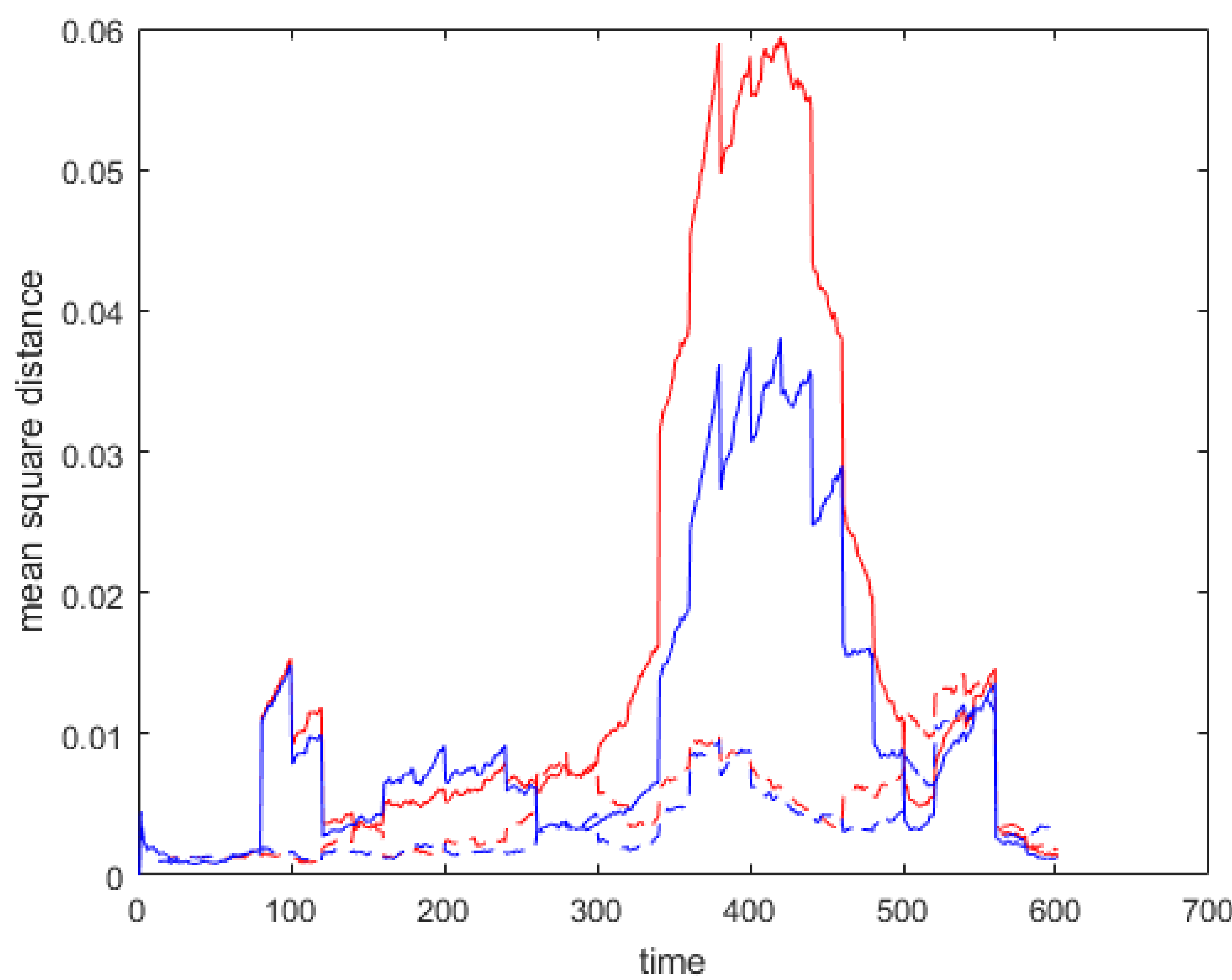

Figure 1: Experiment 10 Type 1. Red: classic SEIK, blue: modified SEIK. The dashed line is the hidden variable, while the other is the assimilated one.

\section{Conclusions}

The modified SEIK filter has obtained better results then the classic SEIK filter in 36 of 40 tests made. This confirms that using the PCA to change the error basis when needed helps significantly to achieve better precision, without rising the number of ensemble elements.

\section{Forthcoming Research}

Testing the new Kalman scheme on a more complicated model, like the MedBFM System, will be a priority. Implementation and optimization will follow up, if the performances will be confirmed.

In the near future, how the candidates for the substituted basis vectors are chosen is a point that will be better investigated, in order to improve further the skill of the scheme.

\section{References}

[1] The Biogeochemical Flux Model (BFM), Equation Description and User Manual, BFM version 5.1, Re lease 1.1, August 2015.

[2] G. Cossarini, P. Lazzari, and C. Solidoro. Spatiotemporal variability of alkalinity in the mediterranean sea Biogeosciences, 12(6), 2015.

3] S. Dobricic and N. Pinardi. An oceanographic three-dimensional variational data assimilation scheme. Ocean Modelling, 2008.

[4] N. Halko, P. G. Matinsson, and J. A. Tropp. Finding structure with randomness: Probabilistic algorithms for constructing approximate matrix decompositions. SIAM Rev., 53(2):217-288, 2011

5] P. Lazzari, C. Solidoro, V. Ibello, S. Salon, A. Teruzzi, K. Béranger, S. Colella, and A. Crise. Seasona and inter-annual variability of plankton chlorophyll and primary production in the mediterranean sea: a modelling approach. Biogeosciences, 9, 2012

[6] P. Lazzari, A. Teruzzi, S.a Salon, S. Campagna, C. Calonaci, S. Colella, M. Tonani, and A. Crise. Preoperational short-term forecasts for the mediterranean sea biogeochemistry. Ocean Science, 6, 2010.

[7] L. Nerger and W. W. Gregg. Assimilation of seawifs data into a global ocean-biogeochemical model using a local seik filter. J. Mar. Syst., 68, 2007.

[8] A. Teruzzi, S. Dobricic, C. Solidoro, and G. Cossarini. A 3-d variational assimilation scheme in coupled transport-biogeochemical models: Forecast of mediterranean biogeochemical properties. J. Geophys. Res. Oceans, 119, 2014

[9] G. Triantafyllou, I. Hoteit, and G. Petihakis. A singular evolutive interpolated kalman filter for efficient data assimilation in a 3-d complex physicalbiogeochemical model of the cretan sea. J. Mar. Syst., 41, 2002. 